Proceedings of the II Symposium on Applied Nuclear Physics and Innovative Technologies, Kraków, September 24-27, 2014

\title{
Project of the Underwater System for Chemical Threat Detection
}

\author{
M. Silarski*, D. Hunik, P. Moskal, M. Smolis, S. Tadeja \\ Institute of Physics, Jagiellonian University, St. Łojasiewicza 11, 30-348 Kraków, Poland
}

\begin{abstract}
In this article we describe a novel method for the detection of explosives and other hazardous substances in the marine environment using neutron activation. Unlike the other considered methods based on this technique we propose to use guides for neutron and gamma quanta which speeds up and simplifies identification. Moreover, it may provide a determination of the density distribution of a dangerous substance. First preliminary results of Monte Carlo simulations dedicated for design of a device exploiting this method are also presented.
\end{abstract}

DOI: 10.12693/APhysPolA.127.1543

PACS: 28.41.Ak, 89.20.Dd, 28.20.Cz, 28.20.Fc

\section{Introduction}

During both World Wars the Baltic Sea was a scene of intense naval warfare and became a "container" for almost whole Nazi chemical munition arsenal. In accordance with the provisions of the Potsdam Conference, Germany was demilitarized but only a small part of their arsenal was neutralized on land. Technologies for safe and effective disposal of chemical weapons were not known shortly after the war. Thus, till 1948 about 250000 tons of munition, including up to 65000 tons of chemical agents, were sunk in Baltic Sea waters by both Germans and Allies. The main known contaminated areas are Little Belt, Bornholm Deep (east of Bornholm) and the southwestern part of the Gotland Deep [1]. Apart from known underwater stockyard there is unknown amount of dangerous war remnants spread over the whole Baltic, especially along maritime convoys paths and in vicinity of coasts. It is not clear how dangerous are these underwater arsenals. At the bottom of the sea (in approximately $5-7^{\circ} \mathrm{C}$ ) the chemical agents take a form of oily liquids hardly soluble in water. Thus, the sunk ammunition does not release hazardous substances. It becomes dangerous however, if the rusted tanks and shells are raised from the bottom of the sea. Chemical munitions, containing mostly mustard gas, was fished several times by fishermen on the Baltic Sea over the last fifty years. Moreover, already in 1952 and 1955 the contamination was found at the polish coast causing serious injuries to people. It was estimated that if only $1 / 6$ of the sunk chemical agents was released into Baltic the life in the sea and at its shores would be entirely ruined for the next 100 years [1]. High economic and environmental costs have been preventing so far any activities aiming at extraction of these hazardous substances, but it is clear that we are about to face a very serious problem in the Baltic Sea. Appropriate actions for preventing the ecological catastrophe

* corresponding author; e-mail: michal.silarski@uj.edu.pl demand a precise knowledge of location and amount of sunk munitions.

Presently used methods for underwater munition detection is based on sonars which show only a shape of underwater objects, like e.g. sunk ships or depth charges. To estimate the amount of dangerous substances and to determine the exact location of sunk munition it is still necessary that people are diving and searching the bottom of the sea. This operation is always very dangerous for divers since the corrosion state of the shells is usually not known. Moreover, these methods are very expensive and slow, thus they cannot be used in practice to search big sea areas. The above mentioned disadvantages can be to large extend overcome by using devices based on neutron activation analysis (NAA) techniques which will be discussed in more details in next sections of this article.

\section{Underwater detection of hazardous substances with neutron beams}

Most of the commonly used explosives or drugs are organic materials. Therefore, they are composed mostly of oxygen, carbon, hydrogen, and nitrogen. War gases contain also sulfur, chlorine, phosphorus and fluorine. Thus, these substances can be unambiguously identified by the determination of the ratio between number of $\mathrm{C}, \mathrm{H}, \mathrm{N}$, $\mathrm{O}, \mathrm{S}, \mathrm{P}$, and $\mathrm{F}$ atoms in a molecule, which can be done noninvasively applying NAA techniques. The suspected item can be irradiated with a flux of neutrons produced using compact generators based e.g. on deuteron-tritium fusion, where deuterons are accelerated to the energy of $0.1 \mathrm{MeV}$ and hit a solid target containing tritium. As a result of the fusion an alpha particle is created together with the neutron, which is emitted nearly isotropically with a well defined energy equal to about $14.1 \mathrm{MeV}$ [2].

Such energy is sufficient to excite all nuclei composing organic substances and the resulting $\gamma$ quanta from the de-excitation of nuclei are then detected by e.g. a germanium detector providing a very good energy resolution. Counting the number of gamma quanta emitted by nuclei from the examined item provides information about its 
stoichiometry. Devices using NAA to detect explosives on the ground were already designed and are produced, e.g. in USA [3] and Poland [4].

In the aquatic environment however we encounter serious problems since neutrons are strongly attenuated by water. Moreover, as in the case of ground detectors, an isotropic generation of neutrons induces a large environmental background, in this case from oxygen. This noise can be significantly reduced by the requirement of the coincident detection of the alpha particle, which allows for the neutron tagging [5]. The attenuation of neutrons can be compensated by decreasing the distance between generator and examined item [6]. There are also solutions based on low energy neutrons which are moderated in water before reaching the tested object. The detector is then counting the gamma quanta from thermal neutron capture and secondary neutrons originating from the irradiated object. The identification is done by searching for anomalies in the observed spectra of gamma quanta and neutrons [7].

However, these methods do not allow to detect explosives buried deeply in the bottom of the sea. Moreover, the device has to approach the suspected item very close and the strong attenuation of neutrons and gamma quanta significantly increases the exposure time and make the interpretation of results difficult. Therefore, we propose to build a detector which uses NAA technique and special guides for neutrons and emitted gamma rays [8].

The device allows for detection of dangerous substances hidden deep in the bottom of the sea with significantly reduced background and provides determination of the density distribution of the dangerous substance in the tested object.

\section{Concept of using the neutron guides}

Scheme of the proposed device is presented in Fig. 1. Neutron generator collides deuterium ions with a tritium target producing a neutron and an $\alpha$ particle. Because of the much higher energy released in this reaction compared to the energy of deuterium, both particles are produced almost isotropically and move back-toback. The $\alpha$ particle is detected by a system of position sensitive detectors, e.g. silicon pads or scintillation hodoscope, disposed on the walls of the generator. Neutrons emitted towards the subject of interrogation fly inside a guide built out of a stainless steal pipe filled with low pressure air or some other gas having low cross-section for neutron interaction. Neutrons after leaving a guide may be scattered inelastically on atomic nuclei in the tested substance. The nuclei deexcite and emit gamma quanta with energy specific to the element. Part of the emitted $\gamma$ quanta fly towards a dedicated detector within an analogous guide which decrease their absorption and scattering with respect to gamma quanta flying in water. The $\gamma$ ray detector could be again a position sensitive detector measuring the energy, time and impact point

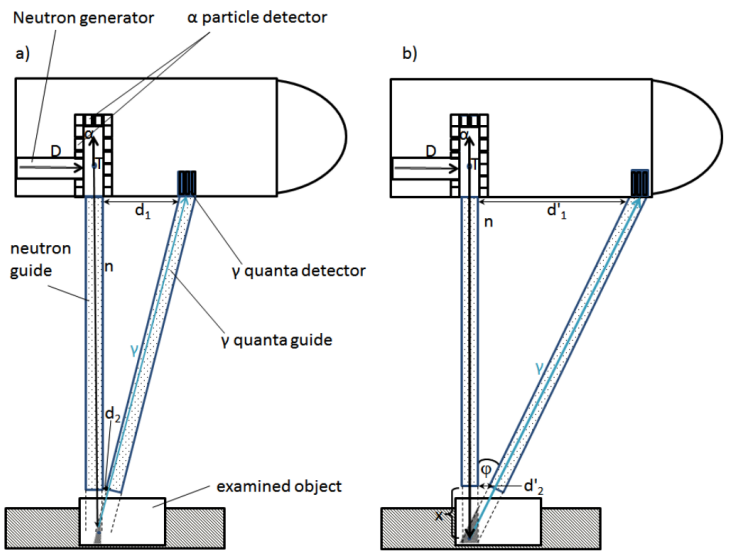

Fig. 1. (a) Schematic view of a device for underwater detection of hazardous substances using neutron beams. Application of guides reduce the scattering of both neutrons and $\gamma$ quanta, while registration of an $\alpha$ particle in anti-coincidence with gamma quanta detector allows to reduce background. (b) Demonstration of how changing of the relative position between neutron and $\gamma$ quanta guides provides a tomographic image of the interrogated object.

of impinging particles. If the diameter-to-length ratio of both guides is small the depth at which $\gamma$ quanta excite nuclei can be determined by measuring the time $\Delta t$ elapsed from the $\alpha$ particle registration until the time of the $\gamma$ quantum registration. Generated neutrons travel with known velocity $v_{\mathrm{n}}$ the distance $L_{\mathrm{n}}$ from the tritium target to the point of interaction. Similarly the gamma emitted from the tested object fly over a distance $L_{\gamma}$ with a speed of light $c$. Thus, $\Delta t$ can be expressed as

$$
\Delta t=L_{\mathrm{n}} / v_{\mathrm{n}}+L_{\gamma} / c-L_{\alpha} / v_{\alpha}
$$

If we know the positions of the generator target and $\gamma$ ray detector and lengths of both guides the distance $x$ covered by neutrons from the end of the guide to the point of interaction can be calculated as

$$
x=\left(\Delta t+\frac{L_{\alpha}}{v_{\alpha}}-\frac{l_{\mathrm{n}}}{v_{\mathrm{n}}}-\frac{l_{\gamma}}{c}\right) \frac{c v_{\mathrm{n}} \cos \phi}{c \cos \phi+v_{\mathrm{n}}},
$$

where $l_{\mathrm{n}}$ and $l_{\gamma}$ are the length of guides for neutrons and $\gamma$ quanta, respectively. Additional information on the depth of interaction is given by changing the relative position of the guides and the angle $\phi$ between them. As it is demonstrated in Fig. 1a and b changing the distance between the guides provides registration of gamma quanta emitted from different parts of the object. This allows one to determine the density distribution of elements building the suspected object. In order to remove background resulting from interaction of neutrons emitted in other directions only signals registered by the $\gamma$ quanta detector in coincidence with signals from $\alpha$ particle detectors mounted in-line with the neutron guide are saved, while the other coincidences are discarded.

Taking into account cross-section for neutron inelastic scattering with different nuclides and the detection efficiency of $\gamma$ quanta we can reconstruct the number of atoms of each element building the object and compare 
them with the known stoichiometry of hazardous substances stored in the database. The whole detection unit can be mounted on a small submarine steered remotely from a ship.

\section{Prototype design: simulations}

In order to optimize the dimensions and relative positions of detectors and guides we have developed dedicated open source software package written in the $\mathrm{C}++$ programming language and based on the Monte Carlo simulation methods. Our goal is to create a fast and user-friendly tool using novel methods of geometry definition and particle tracking based on hypergraphs [9]. The simulation framework is written using the $\mathrm{C}++11$ standard and Open MPI library [10] supporting parallel computing and it is destined for Unix-like operating systems. The application needs to be configured with input file defining scene description (location and shape of all objects included in simulation, as well as substances building them), and neutron source parameters (location, number of generated neutrons and their energies). The parameters of neutron interaction with selected nuclei, e.g. total cross-sections, neutron and $\gamma$ quanta angular distributions and multiplicities, are parameterized as a function of neutron energy using data from the ENDF database [11].

Similarly, gamma quanta energies were taken from the evaluated nuclear structure data files (ENSDF) [12].

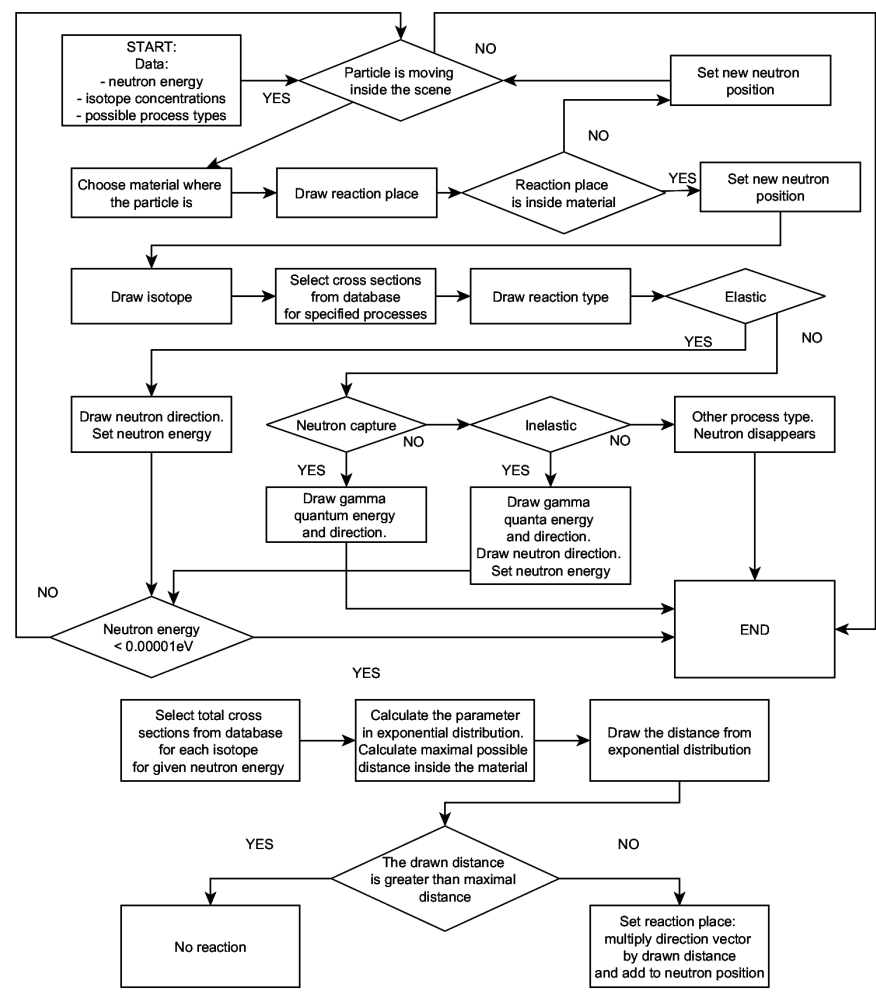

Fig. 2. Scheme of the algorithm used for neutron tracking (top) and details of the determination of neutron reaction place (bottom).
This information is stored in an SQLite database and recalled during the simulation only for elements specified by user. So far we have implemented only main processes induced by $14.1 \mathrm{MeV}$ neutrons which we are interested in, i.e. elastic and inelastic scattering and radiative capture, but in the near future the simulations will be supplemented with other processes, e.g. fission. At present neglected processes are taken into account effectively as one process after which neutron is no longer tracked. Crosssection for this process is calculated in a way that the sum of all processes induced by neutron is equal to the total cross-section for a given nuclei with which the reaction occurred. Neutrons are tracked according to the algorithm presented in Fig. 2 until they reach the scene boundary or their energy goes below the lowest included in the database ${ }^{\dagger}$, i.e. $10^{-5} \mathrm{eV}[11]$. The reaction place is randomly generated with exponential probability distribution taking into account total cross-section values from the database and concentrations of all the isotopes building the substance ${ }^{\ddagger}$. User can choose to calculate concen-
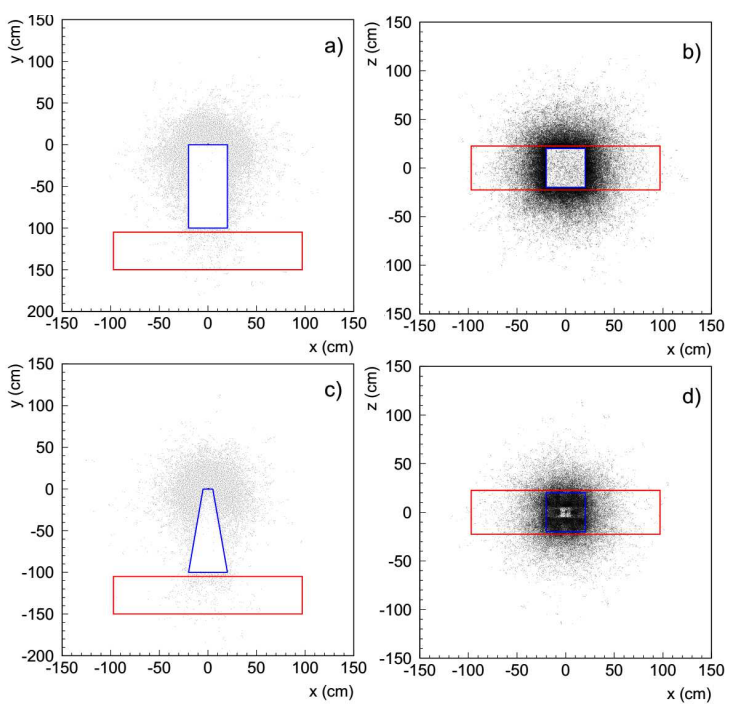

Fig. 3. Distribution of neutron interaction points in $x-y$ view for guide in a shape of (a) cuboid and (b) truncated pyramid. The point-like source of neutrons is located at the origin of the reference frame. The neutron guide is presented as the blue rectangle (a) or trapezoid (c) aligned along the $y$ axis. The container with explosives is represented by the red cuboid aligned horizontally. For better visibility of the influence of neutron guide plots (a), (c) and (b), (d) are made only for $-5 \leq z \leq 5$ and $y \leq 0$, respectively.

trations for a single chemical compound or a mixture of substances for a specified density. Selection of an isotope with which the interaction took place is also random and

$\dagger$ All results presented in this article were obtained however neglecting neutrons with energy $E<10 \mathrm{keV}$.

$\ddagger$ Currently we take into consideration only the most abundant isotope for each element. 
it is done again based on neutron total cross-sections and known stoichiometry of the material in which the reaction is simulated. Next the reaction type is drawn according to cross-section values for each process from the list specified by user in the input file. The direction of neutron after the reaction is generated using angular distributions parametrized with the Legendre polynomials or using uniform distribution if there is no relevant data in the database. It is first determined in the center of mass system and then the four-momentum is transformed back to the laboratory coordinate system. In case of inelastic scattering neutron energy in the laboratory is calculated taking into account the nuclei excitation energy. In the current version of simulations we take into account up to tenth excited nuclei level. Directions of gamma quanta coming from the nuclei deexcitation are currently generated uniformly in the laboratory frame.
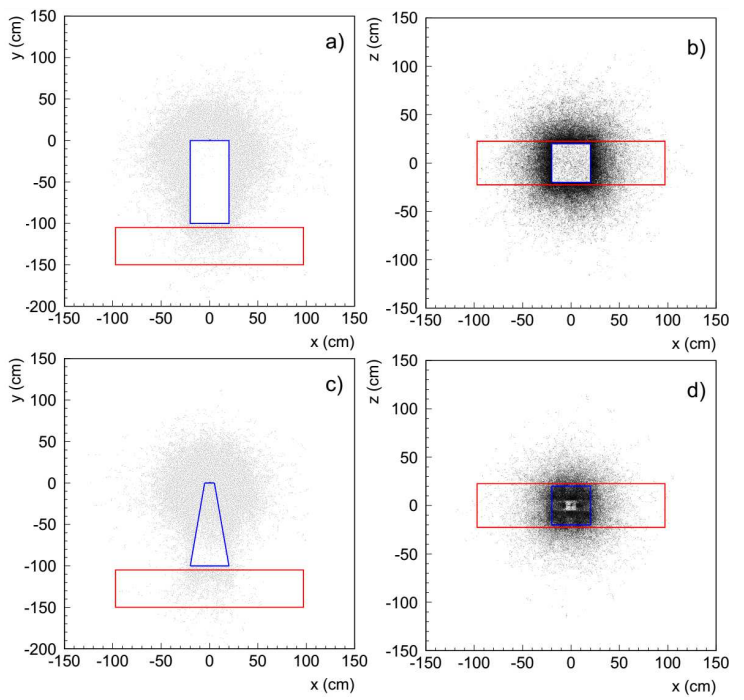

Fig. 4. Distribution of neutron interaction points in $x-y$ view for guide in a shape of (a) cuboid and (b) truncated pyramid. The point-like source of neutrons is located at the origin of the reference frame. The neutron guide is presented as the blue rectangle (a) or trapezoid (c) aligned along the $y$-axis. The container with explosives is represented by the red cuboid aligned horizontally. For better visibility of the influence of neutron guide plots (a), (c) and (b), (d) are made only for $-20 \leq z \leq 20$ and $y \leq 0$, respectively.

As a starting point for design of the device for underwater threats detection we have defined a simple setup with point-like source generating $14.1 \mathrm{MeV}$ neutrons uniformly in space. The scheme of the simulated setup with superimposed points of of neutrons interaction are shown in Figs. 3 and 4 . We have considered two neutron guides filled with air under normal conditions: cuboid with dimensions $40 \times 40 \times 100 \mathrm{~cm}^{3}$ (Figs. 3a and $4 \mathrm{a}$ ) and truncated pyramid with height equal to $100 \mathrm{~cm}$ and bases with dimensions of $5 \mathrm{~cm}$ and $20 \mathrm{~cm}$ (Figs. 3b and $4 \mathrm{~b}$ ). The interrogated object with dimensions $194 \times 255 \times 50 \mathrm{~cm}^{3}$ lies on the bottom of a sea and contains mustard gas. As one can see in Fig. 5 the dis- tribution of the path length of neutrons in water is characterized by a mean free path of about $9.5 \mathrm{~cm}$. At the same time the flux flying through the guide filled with air reaches the container with mustard gas and excites its nuclei. Comparing Fig. 3a and b one can see also that both shapes of the neutron guide give effectively the same flux irradiating the gas container, but for the trapezoidal shape a better spatial separation between regions where neutrons interact in water and in the interrogated object is clearly visible. The optimization of shapes and configuration of the neutron and gamma quanta guides will be a subject of future investigations.

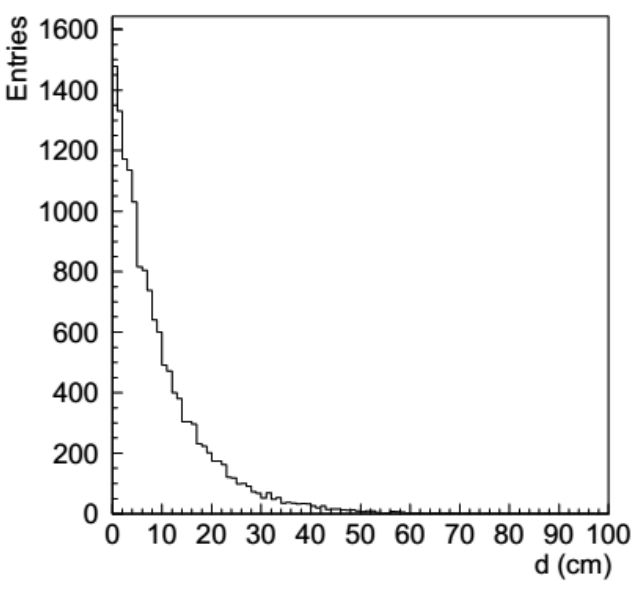

Fig. 5. Distribution of the distance covered by neutron till the point of first interaction in the water (for $y \geq 0$ ).

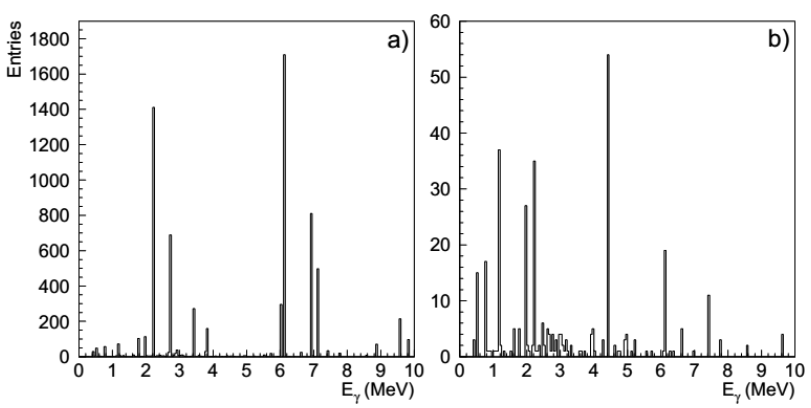

Fig. 6. Distributions of energies of gamma quanta coming from nuclei excitation in (a) water and (b) mustard gas.

The energy spectra of gamma quanta from deexcitation of nuclei in water and interrogated object are presented in Fig. 6. In Fig. 6a one can see strong lines from the excitation of oxygen with energies about $3 \mathrm{MeV}, 6 \mathrm{MeV}$ and $7 \mathrm{MeV}$, and characteristic line of neutron capture by hydrogen (about $2.2 \mathrm{MeV}$ ). The spectrum for the container with mustard gas in Fig. 6b contains a big peak for gamma quanta emitted by carbon ${ }^{12} \mathrm{C}\left(E_{\gamma}=4.43 \mathrm{MeV}\right)$ and a structure at low energies and a peak at $E_{\gamma} \approx 6 \mathrm{MeV}$ coming from chlorine ${ }^{37} \mathrm{Cl}$. Hydrogen and sulfur ${ }^{32} \mathrm{~S}$ compose one line at $E_{\gamma}=2.2 \mathrm{MeV}$, which shows that identification of this element will be difficult. 


\section{Summary and outlook}

Methods of chemical threat detection based on neutron activation have a huge potential and may open a new frontier in homeland security. In the aquatic environment application of this method encounters serious problems since neutrons are strongly attenuated. In order to suppress this attenuation and to decrease background from gamma radiation induced in the water we propose to use guides for neutrons and gamma quanta which speeds up and simplifies identification. Moreover, it may provide a determination of the density distribution of a dangerous substance. For designing of a device exploiting this idea we have been developing a fast and user-friendly simulation package using novel methods of geometry definition and particle tracking based on hypergraphs. Although we are in a very early stage of the development the first results indicate that indeed the guides will increase the performance of underwater threats detection with fast neutrons.

\section{Acknowledgments}

This work was supported by the Polish Ministry of Science and Higher Education through grant No. 7150/E$338 / \mathrm{M} / 2014$ for the support of young researchers and Ph.D. students of the Department of Physics, Astronomy and Applied Computer Science of the Jagiellonian University.

\section{References}

[1] T. Kasperek, Czas Morza 1, 15 (2001).

[2] P. Moskal, Ann. UMCS Physica 66, 71 (2012).

[3] B.C. Maglich, AIP Conf. Proc. 796, 431 (2005).
[4] Ł. Kaźmierczak, S. Borsuk, M. Gierlik, Z. Guzik, J. Iwanowska-Hanke, S. Korolczuk, T. Kozłowski, T. Krakowski, R. Marcinkowski, L. Swiderski, M. Szeptycka, J. Szewiński, A. Urban, Acta Phys. Pol. A 127, 1540 (2015).

[5] M. Silarski, Acta Phys. Pol. B Suppl. 6, 1061 (2013).

[6] C. Eleon, B. Perot, C. Carasco, D. Sudac, J. Obhodas, V. Valkovic, Nucl. Instrum. Methods Phys. Res. A 629, 220 (2011).

[7] D. Lambertus, T. Schneider-Pungs, K. Buckup, Patent application WO2012089584 A1.

[8] M. Silarski, P. Moskal, Patent application PL409388.

[9] E. Grabska, A. Łachwa, G. Ślusarczyk, Adv. Eng. Inform. 26, 681 (2012).

[10] M.J. Quinn, Parallel Programming in C with MPI and OpenMP, McGraw-Hill, 2004.

[11] M.B. Chadwick, M. Herman, P. Obložinský, M.E. Dunn, Y. Danon, A.C. Kahler, D.L. Smith, B. Pritychenko, G. Arbanas, R. Arcilla, R. Brewer, D.A. Brown, R. Capote, A.D. Carlson, Y.S. Cho, H. Derrien, K. Guber, G.M. Hale, S. Hoblit, S. Holloway, T.D. Johnson, T. Kawano, B.C. Kiedrowski, H. Kim, S. Kunieda, N.M. Larson, L. Leal, J.P. Lestone, R.C. Little, E.A. McCutchan, R.E. MacFarlane, M. MacInnes, C.M. Mattoon, R.D. McKnight, S.F. Mughabghab, G.P.A. Nobre, G. Palmiotti, A. Palumbo, M.T. Pigni, V.G. Pronyaev, R.O. Sayer, A.A. Sonzogni, N.C. Summers, P. Talou, I.J. Thompson, A. Trkov, R.L. Vogt, S.C. van der Marck, A. Wallner, M.C. White, Nucl. Data Sheets 112, 2887 (2011)

[12] Data extracted using the NNDC On-Line Data Service from the ENSDF database; M. R. Bhat, Nuclear Data for Science and Technology, Ed. S.M. Qaim, Springer, Berlin 1992, p. 817. 